Anestesiología

Enero-Marzo 2022

Vol. 45. No. 1. pp 35-39

doi: $10.35366 / 102901$

\section{Un modelo para la evaluación de las habilidades no técnicas en anestesiología: una revisión de la literatura}

\author{
A model for the assessment of non-technical skills \\ in anesthesiology: a literature review
}

\author{
Dr. Ulises Sánchez-Vásquez,* Dr. Juan Sebastián Espino-Núñez,* \\ Dra. Andrea Figueroa-Morales, * Dr. Rodrigo Rubio-Martínez ${ }^{\ddagger}$
}

Citar como: Sánchez-Vásquez U, Espino-Núñez JS, Figueroa-Morales A, Rubio-Martínez R. Un modelo para la evaluación de las habilidades no técnicas en anestesiología: una revisión de la literatura. Rev Mex Anestesiol. 2022; 45 (1): 35-39. https://dx.doi.org/10.35366/102901

\begin{abstract}
RESUMEN. Hasta 80\% de los errores médicos se deben a fallas en factores humanos (mala comunicación, monitoreo inadecuado, fallas de verificación, etc.), por lo que el entrenamiento de los anestesiólogos exige el desarrollo de habilidades no técnicas en anestesiología. Las habilidades no técnicas son las habilidades cognitivas, sociales y personales que complementan las habilidades técnicas, y que contribuyen al desempeño seguro y eficiente de la tarea. En 2004 la Universidad de Aberdeen fue la primera en plantear un modelo para la definición y evaluación de estas habilidades en el ámbito médico. El modelo práctico consta de 15 elementos incluidos en cuatro categorías: manejo de la tarea, trabajo en equipo, conciencia de la situación y toma de decisiones. La herramienta es utilizada por anestesiólogos graduados para evaluar a quienes están en entrenamiento en el quirófano o mediante simulación clínica. La validez de este sistema, así como su importancia en la seguridad del paciente, ha sido demostrada por diferentes estudios.
\end{abstract}

ABSTRACT. Close to $80 \%$ of medical errors are due to human factors (poor communication, inadequate monitoring, failure to check, etc.), which is why training for anaesthetists requires developing essential soft skills for Anaesthesiology. Soft skills are defined as specific cognitive, socio-emotional and interpersonal abilities complementing core skills which contribute to the safe and efficient carrying out of a job-specific task. In 2004, the University of Aberdeen established a first model for defining and evaluating these soft skills. The model consists of 15 elements across four categories: task management, team working, situational awareness and decision-making. The model is a tool employed by postgraduate anaesthesiologists to assess trainees in the operating theatre or through clinical simulation. The validity of this system, as well as its importance for patient safety have been demonstrated in a range of studies.

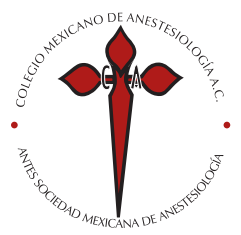

Palabras clave: Habilidades no técnicas, errores médicos, factor humano, sistema Anaesthetists' non-technical skills, simulación clínica.

Keywords: Soft skills, medical errors, human factors, Anaesthetists' non-technical skills system, clinical simulation.

* Anestesiólogo. Algólogo. ‡ Anestesiólogo. Coordinador de Anestesia. Centro de Simulación.

Centro Médico ABC, CDMX, México.

Correspondencia: Dr. Ulises Sánchez-Vásquez The American British Cowdray, Medical Center, IAP.

Sur 136 Núm. 116, Col. Las Américas, 01120, Alcaldía Álvaro Obregón, Ciudad de México, Tel: (55) 4953-2782

E-mail: ulises.sanchez. vasquez@gmail.com

Siglas:

ANTS = Anaesthetists' non-technical skills.

Recibido: 16-07-2019

Aceptado: 04-09-2019

\section{INTRODUCCIÓN}

D e acuerdo con el Instituto de Medicina de Estados Unidos, de 44,000 a 98,000 personas mueren cada año debido a errores médicos ${ }^{(1)}$. Investigaciones de causa raíz han reportado que hasta $80 \%$ de estos errores son el resultado de fallas en factores humanos como mala comunicación, monitoreo inadecuado, fallas de verificación de medicamentos y de equipo, etc. ${ }^{(2)}$. El currículo médico y de las diferentes especialidades médicas se ha enfocado a lo largo de los años en el desarrollo cognitivo y técnico de los alumnos; sin embargo, las demandas de la atención de salud exigen el desarrollo de competencias no técnicas ${ }^{(3-6)}$.
Esta revisión se centra en la definición de las habilidades no técnicas basadas en el modelo ANTS con la intención de que sea de utilidad para el lector en la evaluación de los médicos en entrenamiento a su cargo o como herramienta de metacognición para mejorar su práctica diaria.

La anestesiología es una especialidad que involucra riesgos para el paciente que pueden convertirse en escenarios de alta intensidad desde el inicio hasta la finalización de las tareas, y exige al anestesiólogo el monitoreo continuo y la respuesta rápida a eventos críticos. La resolución exitosa del caso requiere de habilidades técnicas y no técnicas, de las cuales las últimas no habían sido claramente definidas ${ }^{(7)}$. El entrenamiento de los médicos anestesiólogos sigue demostrando una falta de 
concordancia entre el desarrollo de las habilidades técnicas y las no técnicas ${ }^{(8)}$.

\section{HABILIDADES NO TÉCNICAS EN ANESTESIOLOGÍA}

En 2004 la Universidad de Aberdeen planteó un sistema para la definición y evaluación de las habilidades no técnicas en anestesiología (Anaesthetists’ Non-Technical Skills, ANTS). Las habilidades no técnicas se definen como «las habilidades cognitivas, sociales y personales que complementan las habilidades técnicas, y que contribuyen al desempeño seguro y eficiente de la tarea» ${ }^{(9)}$.

\section{SISTEMA ANTS}

El sistema ANTS es un conjunto de marcadores conductuales desarrollado por psicólogos industriales y anestesiólogos durante un proyecto colaborativo que duró cuatro años. Describe las principales habilidades no técnicas observables asociadas a una buena práctica anestésica y proporciona un marco teórico y una herramienta de evaluación. El sistema ANTS ofrece un lenguaje para la discusión de «aspectos conductuales» del rendimiento en un modelo jerárquico de tres niveles. El nivel más alto consta de cuatro categorías, dentro de las cuales se incluyen 15 elementos (Tabla 1). Cada elemento tiene su definición y ejemplos de buena o mala práctica.

\section{CATEGORÍAS DEL SISTEMA ANTS}

Manejo de la tarea: habilidades para organizar las actividades y los recursos, ya sean planes de casos individuales o problemas de programación a largo plazo. Tiene cuatro elementos:
1. Planificar y preparar: desarrollar estrategias primarias y alternativas, en caso de contingencia, revisarlas y actualizarlas si es necesario.

2. Priorizar: enumerar según su importancia las tareas, actividades, problemas, canales de información, etc.; ser capaz de identificar problemas clave y asignarles la atención que requieran, y evitar distraerse con asuntos menos importantes o irrelevantes.

3. Proporcionar y mantener estándares: respaldar la seguridad y la calidad al adherirse a los principios aceptados de la anestesia; seguir, cuando sea posible, códigos de buena práctica, protocolos o guías de tratamiento y checklists mentales.

4. Identificar y utilizar los recursos: establecer los recursos necesarios y con cuáles se cuentan, usarlos para lograr los objetivos con mínima interrupción, estrés o sobrecarga de trabajo (mental y física) ${ }^{(2)}$.

Trabajo en equipo: habilidades para trabajar en un contexto de grupo, desde cualquier rol, asegurando la finalización de la tarea en conjunto y la satisfacción de cada miembro del equipo. Consta de cinco elementos:

1. Coordinar actividades con los miembros del equipo: trabajar en conjunto tanto para actividades físicas como cognitivas. Comprender los roles y las responsabilidades de los diferentes miembros del equipo, y garantizar que se emplee un enfoque de colaboración.

2. Intercambiar información: dar y recibir conocimiento e información necesaria para la coordinación del equipo y la finalización de la tarea.

3. Usar la autoridad y asertividad: dirigir al equipo y/o la tarea aceptando un rol distinto al de líder cuando sea apropiado; adoptar una forma convenientemente enérgica para tocar un punto, y adaptarlo al equipo y/o a la situación.

Tabla 1: El sistema Anaesthetists' non-technical skills.

Categoría: manejo de la tarea

Elementos:
1. Planear y preparar
2. Priorizar
3. Proporcionar y mantener los estándares
4. Identificar y utilizar los recursos

Categoría: conciencia de la situación
Categoría: trabajo en equipo

Elementos:

5. Coordinar actividades con los miembros del equipo

6. Intercambiar información

7. Usar la autoridad y asertividad

8. Evaluar las capacidades

9. Apoyar a los otros

Categoría: toma de decisiones
Elementos:

10. Recopilar información

11. Reconocer y entender

12. Anticiparse
Elementos:

13. Identificar otras opciones

14. Balancear riesgos y seleccionar opciones

15. Reevaluar

El sistema Anaesthetists' non-technical skills consta de cuatro categorías que incluyen 15 elementos. 
4. Evaluar las capacidades de cada miembro: juzgar las habilidades de los diferentes miembros del equipo y su capacidad para manejar una situación; estar alerta a los factores que puedan limitar su capacidad de desempeñarse efectivamente (por ejemplo, el nivel de experiencia, estrés, fatiga).

5. Apoyar a los demás: proporcionar ayuda física, cognitiva o emocional a otros miembros del equipo ${ }^{(2)}$.

Es el rubro con mayor evidencia sobre su importancia en el quirófano y su impacto en la seguridad del paciente ${ }^{(10,11)}$. Se han identificado tres tipos principales de intervención eficaces para mejorar el trabajo en equipo en el quirófano: métodos estructurados para compartir información (por ejemplo, listas de verificación), capacitación del equipo in situ y ajustes organizacionales $^{(12,13)}$. Los anestesiólogos pueden desempeñar un papel importante en el mantenimiento de la armonía y el rendimiento del equipo mediante la comunicación verbal y la no verbal ${ }^{(14-16)}$.

Conciencia de la situación: habilidades necesarias para desarrollar y mantener un conocimiento general del entorno basado en la observación de todos los aspectos relevantes del quirófano (paciente, equipo humano y técnico, tiempo, monitoreo); entendiendo lo que significan, y anticipándose a lo que podría pasar. Consta de tres elementos:

1. Recopilar información (percepción): consiste en reunir información de forma activa y específica mediante observación continua y el monitoreo de todas las variables disponibles así como verificar dicha información para confirmar su fiabilidad (es decir, que no sean artefactos).

2. Reconocer y entender (comprensión): interpretar la información recopilada (con respecto a modelos mentales existentes) para identificar la coincidencia o desajuste entre la situación y el estado esperado.

3. Anticiparse (proyección): básicamente responde a «qué pasa si...», responde al futuro sobre posibles resultados y visualiza las consecuencias de las acciones, intervenciones o las no intervenciones. Ejecuta proyecciones de la situación actual para predecir lo que podría suceder en un futuro cercano ${ }^{(2)}$.

El anestesiólogo necesita distribuir su atención a través de varias fuentes de información. Esta conciencia debe ser compartida con los miembros del equipo. Tanto las interrupciones como las distracciones han sido señaladas como disruptores comunes de la conciencia situacional y pueden representar riesgos para la memoria prospectiva (recordar cosas que hacer en el futuro, por ejemplo, readministrar un medicamento en 10 minutos $)^{(17,18)}$. Schulz y colaboradores encontraron que entre 2002-2013 en 74\% de las demandas a anestesiólogos sucedió un error en esta categoría que contribuyó a la muerte del paciente o a daño cerebral. Los errores de percepción fueron los más comunes (42\%), mientras que los de comprensión (29\%) y proyección (29\%) fueron menos frecuentes ${ }^{(19)}$.

Toma de decisiones: es un proceso cognitivo para llegar a un juicio, seleccionar una opción y elegir qué acción tomar para satisfacer las necesidades de una situación dada tanto en condiciones electivas como en situaciones de emergencia. Consta de tres elementos:

1. Identificar otras opciones: generar y considerar posibilidades o cursos de acción alternativos al tomar la decisión o al resolver un problema.

2. Equilibrar riesgos y seleccionar opciones: evaluar los riesgos o los beneficios de una situación, considerando las ventajas y desventajas de los diferentes cursos de acción; elegir una solución o un curso de acción basado en este proceso.

3. Reevaluar: revisar continuamente la idoneidad de las opciones identificadas, evaluadas y seleccionadas; $\mathrm{y}$ reevaluar la situación después de la implementación de una acción determinada.

En anestesiología hay un ciclo continuo de monitoreo y reevaluación del entorno, y luego se toman las medidas apropiadas. Los principales tipos de toma de decisiones son: el reconocimiento mejorado (reconocimiento de patrones o proceso intuitivo), el basado en reglas, el analítico (por ejemplo, comparar distintos cursos de acción) y el creativo ${ }^{(7)}$. Además, es importante mencionar que la toma de decisiones varía de acuerdo con las condiciones del caso o del entorno, por ejemplo, la presión del tiempo, la complejidad de la tarea, la viabilidad de las opciones y el nivel de restricción, el apoyo y los recursos existentes ${ }^{(14)}$.

\section{IMPLEMENTACIÓN DEL SISTEMA ANTS}

La herramienta está diseñada para ser utilizada por anestesiólogos experimentados para evaluar a otros que estén en entrenamiento y que apenas hayan alcanzado las competencias técnicas básicas ${ }^{(9)}$. Las calificaciones son hechas donde sucede el procedimiento anestésico, en el quirófano o en las instalaciones del simulador. La evaluación de cada categoría y de cada elemento se basa en la observación del comportamiento del alumno que después será integrada en una escala de cuatro puntos. La validez, confiabilidad y utilidad del sistema ANTS han sido validadas por Fletcher y colaboradores ${ }^{(20)}$. La importancia de una escala estandarizada para la definición y evaluación de las habilidades no técnicas se puede apreciar en un estudio previo por Byrne y Greaves, en el que se observó una baja concordancia entre evaluadores al calificar habilidades no técnicas ${ }^{(21)}$.

Novenau y su equipo compararon en un estudio la evaluación en simulación de habilidades técnicas y no técnicas entre observadores entrenados y la autoevaluación o evaluación por colegas no entrenados e identificaron una superioridad signi- 
ficativa en la evaluación de observadores entrenados ${ }^{(22)}$. Una revisión sistemática por Davis y colegas volvió a identificar una debilidad en la evidencia existente para la autoevaluación como herramienta evaluativa, por lo que la valoración por observadores externos entrenados puede aumentar la validez de la evaluación ${ }^{(17)}$. Por otro lado, en un estudio por Watkins y colaboradores se comparó a novatos contra expertos como evaluadores de habilidades no técnicas bajo dos metodologías: ANTS y BARS, y encontraron que había mayor correlación interevaluador entre los más novatos que entre los expertos ${ }^{(18)}$. Estos hallazgos probablemente obedecen a una menor flexibilidad para interpretar situaciones por parte de observadores con menos experiencia.

\section{SIMULACIÓN CLÍNICA Y ANTS}

El entrenamiento del modelo ANTS se puede lograr a través de la simulación clínica y es necesario un enfoque en el manejo de recursos en crisis (Crisis Resource Management, CRM) mediante sesiones en aula y escenarios de simulación ${ }^{(23)}$. Está demostrado que la simulación permite la enseñanza y evaluación de las habilidades necesarias para resolver efectivamente una situación de atención médica tanto en habilidades técnicas como en no técnicas ${ }^{(24-28)}$. Las sesiones regulares de simulación tienen mejores resultados que las sesiones aisladas ${ }^{(29,30)}$. Además, el desarrollo de simuladores de alta fidelidad permite la recreación de escenarios clínicos para examinar aspectos conductuales o de comportamiento de manera individual o en equipo ${ }^{(23,31)}$. La simulación también permite realizar un resumen basado en la literatura científica para discutir y proporcionar retroalimentación sobre los aspectos conductuales del desempeño así como para investigar procesos cognitivos subyacentes a través del debriefing ${ }^{(10)}$.

\section{CONCLUSIONES}

El modelo ANTS no es el único que existe para la estandarización de las definiciones; sin embargo, consideramos que es un buen inicio y un sistema práctico para alimentar la conversación profesional en nuestro gremio sobre un tema al que debemos dedicar más recursos. La formación de nuevos anestesiólogos exige más que devorar libros y comprender conceptos fisiológicos, farmacológicos, etc. La formación de profesionales que puedan integrarse de forma interdisciplinaria en la atención de pacientes de forma segura exige la enseñanza y evaluación de habilidades no técnicas.

\section{REFERENCIAS}

1. Institute of Medicine T. To err is human: building a safer health system. 1999. 3 - to err is human - summary.pdf.

2. Flin R, Glavin R, Maran N, Patey R. Framework for observing and rating anaesthetists' non-technical skills team task management decision making situation anaesthetists' non-technical skills (ANTS) System Handbook. V1.0. Aberdeen: University of Aberdeen Press (UniPrint); 2012.

3. Hull L, Arora S, Symons NRA, et al. Training faculty in nontechnical skill assessment. Ann Surg. 2013;258:370-375. doi: 10.1097/ SLA.0b013e318279560b.

4. Reedy GB, Lavelle M, Simpson T, Anderson JE. Development of the human factors skills for healthcare instrument: a valid and reliable tool for assessing interprofessional learning across healthcare practice settings. BMJ STEL. 2017;3:135-141. doi: 10.1136/bmjstel-2016-000159.

5. Sevdalis N, Hull L, Birnbach DJ. Improving patient safety in the operating theatre and perioperative care: obstacles, interventions, and priorities for accelerating progress. Br J Anaesth. 2012;109:i3-i16. doi: 10.1093/bja/aes391.

6. Hull L, Arora S, Symons NRA, et al. Training faculty in nontechnical skill assessment: national guidelines on program requirements. Ann Surg. 2013;258:370-375. doi: 10.1097/SLA.0b013e318279560b.

7. Fletcher GC, McGeorge P, Flin RH, Glavin RJ, Maran NJ. The role of non-technical skills in anaesthesia: a review of current literature. Br J Anaesth. 2002;88:418-429. doi: 10.1093/bja/88.3.418.

8. Gjeraa K, Jepsen RM, Rewers M, Ostergaard D, Dieckmann P. Exploring the relationship between anaesthesiologists' non-technical and technical skills. Acta Anaesthesiol Scand. 2016;60:36-47. doi: 10.1111/aas.12598.

9. Flin R, Patey R, Glavin R, Maran N. Anaesthetists' non-technical skills. Br J Anaesth. 2010;105:38-44. doi: 10.1093/bja/aeq134.

10. Manser T. Teamwork and patient safety in dynamic domains of healthcare: a review of the literature. Acta Anaesthesiol Scand. 2009;53:143-151. doi: 10.1111/j.1399-6576.2008.01717.x.
11. Dietz AS, Pronovost PJ, Mendez-Tellez PA, et al. A systematic review of teamwork in the intensive care unit: what do we know about teamwork, team tasks, and improvement strategies? J Crit Care. 2014;29:908-914. doi: 10.1016/j.jcrc.2014.05.025.

12. Weller J, Boyd M. Making a difference through improving teamwork in the operating room: a systematic review of the evidence on what works. Curr Anesthesiol Rep. 2014;4:77-83. doi: 10.1007/s40140-014-0050-0.

13. Wacker J, Kolbe M. Leadership and teamwork in anesthesia-Making use of human factors to improve clinical performance. Trends Anaesth Crit Care. 2014;4:200-205. doi: 10.1016/j.tacc.2014.09.002.

14. Flin R, Maran N. Basic concepts for crew resource management and non-technical skills. Best Pract Res Clin Anaesthesiol. 2015;29:27-39. doi: 10.1016/j.bpa.2015.02.002.

15. Rutherford JS. Monitoring teamwork: a narrative review. Anaesthesia. 2017;72:84-94. doi: 10.1111/anae.13744.

16. Gordon M, Fell CW, Box H, Farrell M, Stewart A. Learning health "safety" within non-technical skills interprofessional simulation education: a qualitative study. Med Educ Online. 2017;22:1272838. do i:10.1080/10872981.2017.1272838.

17. Davis DA, Mazmanian PE, Fordis M, Harrison R Van, Thorpe KE, Perrier L. Accuracy of physician self-assessment compared a systematic review. JAMA. 2006;296:1094-1102. doi: 10.1001/jama.296.9.1094.

18. Watkins SC, Roberts DA, Boulet JR, McEvoy MD, Weinger MB. Evaluation of a simpler tool to assess nontechnical skills during simulated critical events. Simul Healthc. 2017;12:69-75. doi: 10.1097/SIH.0000000000000199.

19. Schulz CM, Burden A, Posner KL, et al. Frequency and type of situational awareness errors contributing to death and brain damage: A closed claims analysis. Anesthesiology. 2017;127:326-337. doi: 10.1097/ ALN.0000000000001661.

20. Fletcher G, Flin R, McGeorge M, Glavin R, Maran N, Patey R. Anaesthetists' non-technical skills (ANTS): evaluation of a behavioural marker system. Br J Anaesth. 2003;90:580-588. doi: 10.1093/bja/aeg112. 
21. Byrne AJ, Greaves JD. Assessment instruments used during anaesthetic simulation: review of published studies. Br J Anaesth. 2001;86:445450.

22. Noveanu J, Amsler F, Ummenhofer W, von Wyl T, Zuercher M. Assessment of simulated emergency scenarios: are trained observers necessary? Prehosp Emerg Care. 2017;21:511-524. doi:10.1080/10903 127.2017.1302528.

23. Gaba DM, Howard SK, Fish KJ, Smith BE, Sowb YA. Simulationbased training in anesthesia crisis resource management (ACRM): a decade of experience. Simul Gaming. 2001;32:175-193. doi: $0.1177 / 104687810103200206$.

24. Okuda Y, Bryson EO, DeMaria SJ, et al. The utility of simulation in medical education: what is the evidence? Mt Sinai J Med. 2009;76:330343. doi: 10.1002/msj.20127.

25. Leblanc VR. Review article: simulation in anesthesia: State of the science and looking forward. Can J Anesth. 2012;59:193-202. doi: 10.1007/ s12630-011-9638-8.
26. Flin R, Maran N. Identifying and training non-technical skills for teams in acute medicine. Qual Saf Heal Care. 2004;13:180-84. doi: 10.1136/qshc.2004.009993.

27. Doumouras AG, Hamidi M, Lung K, et al. Non-technical skills of surgeons and anaesthetists in simulated operating theatre crises. $\mathrm{Br} \mathrm{J}$ Surg. 2017;104:1028-1036. doi: 10.1002/bjs.10526.

28. Boulet JR, Murray DJ. Simulation-based assessment in anesthesiology. Requirements for practical implementation. Anesthesiology. 2010;112:1041-1052. doi: 10.1097/ALN.0b013e3181cea265.

29. Weinger MB, Burden AR, Steadman RH, Gaba DM. Misconceptions surrounding the maintenance of certification in anesthesiology simulation course. Anesthesiology. 2014;121:655-659.

30. Zausig YA, Grube C, Boeker-Blum T, et al. Inefficacy of simulator-based training on anaesthesiologists' non-technical skills. Acta Anaesthesiol Scand. 2009;53:611-619. doi: 10.1111/j.1399-6576.2009.01946.x.

31. Lewis R, Strachan A, Smith MM. Is high fidelity simulation the most effective method for the development of non-technical skills in nursing? A review of the current evidence. Open Nurs J. 2012;6:82-89. 